\title{
ON THE UNIFORM ERGODIC THEOREM. II
}

\section{MICHAEL LIN ${ }^{1}$}

ABSTRACT. Let $\left\{T_{t}\right\}$ be a strongly continuous semigroup of bounded linear operators on a Banach space $X$, satisfying $\lim _{t \rightarrow \infty}\left\|T_{t}\right\| / t=0$. We prove the equivalence of the following conditions: (1) $t^{-1} \int_{0}^{t} T_{r} d r$ converges uniformly as $t \rightarrow \infty$. (2) The infinitesimal generator $A$ has closed range. (3) $\lim _{\lambda \rightarrow 0^{+}}{ }^{\lambda R_{\lambda}}$ exists in the uniform operator topology.

In [6] the author has proved that for a bounded linear operator $T$ on a Banach space $X$ satisfying $\left\|T^{n} / n\right\| \rightarrow 0, N^{-1} \Sigma_{n=0}^{N-1} T^{n}$ converges uniformly if and only if $(I-T) X$ is closed. In this paper we obtain the analogous result for the continuous case, generalizing some results of Hille and Phillips [5, Theorem 18.8.4] without using the operational calculus devised by Hille. Abel convergence in the discrete case is treated in the appendix.

Theorem. Let $\left\{T_{t}\right\}_{t \geq 0}$ be a strongly continuous semigroup of bounded linear operators with $T_{0}=I$, satisfying $\left\|T_{t} / t\right\| \rightarrow 0$. Let $A$ be the infinitesimal generator of $\left\{T_{t}\right\}$ and let $R_{\lambda} x=\int_{0}^{\infty} e^{-\lambda t} T_{t} x d t$ be the resolvent family $(\lambda>0)$. Then the following conditions are equivalent:

(1) There exists a bounded linear operator $E$ such that $\left\|t^{-1} \int_{0}^{t} T_{s} d s-E\right\|_{t \rightarrow \infty} 0$.

(2) A has closed range.

(3) $N^{-1} \sum_{n=0}^{N-1} R_{1}^{n}$ converges uniformly.

(4) There exists a projection $E$ on $\left\{x: T_{t} x=x, \forall t>0\right\}$ such that $\lim _{\lambda \rightarrow 0^{+}}\left\|\lambda R_{\lambda}-E\right\|=0$.

Lemma 1. Under the assumptions of the theorem, $\lim _{n \rightarrow \infty}\left\|\left(\lambda R_{\lambda}\right)^{n}\right\| / n=0$ for every $\lambda>0$.

Proof. If $t$ is large, $\left\|T_{t}\right\| \leq \epsilon t$ and $w_{0}=\lim _{t \rightarrow \infty} \log \left\|T_{t}\right\| / t \leq$

Received by the editors August 16, 1973.

AMS (MOS) subject classifications (1970). Primary 47A35, 47D05.

Key words and phrases. Ergodic theorem, ergodicity of semigroups, Abel ergodicity.

${ }^{1}$ Research supported by NSF grant GP-34118. 
$\lim t^{-1}(\log t+\log \epsilon)=0$. In [2, Lemma VIII.1.12] it is shown that for $\lambda>0 \geq w_{0}$,

$$
R_{\lambda}^{n} x=(n-1) !^{-1} \int_{0}^{\infty} e^{-\lambda t} t^{n-1} T_{t} x d t .
$$

By induction and integration by parts we have $\left(\lambda^{n} / n !\right) \int_{0}^{\infty} e^{-\lambda t} t^{n} d t=1 / \lambda$.

Fix $\epsilon>0$. Then $\left\|T_{t}\right\| \leq \epsilon t$ for $t \geq t_{0}(\epsilon)$. Since $\left\|T_{t} x\right\|$ is continuous on $\left[0, t_{0}\right]$, it is bounded, and by the principle of uniform boundedness $\left\|T_{t}\right\| \leq K$ for $0 \leq t \leq t_{0}$. Thus

$$
\left\|\left(\lambda R_{\lambda}\right)^{n}\right\| / n \leq \frac{\lambda^{n}}{n !} K \int_{0}^{t_{0}} e^{-\lambda t} t^{n-1} d t+\epsilon \frac{\lambda^{n}}{n !} \int_{t_{0}}^{\infty} e^{-\lambda t} t^{n-1} t d t \leq \frac{K}{n}+\frac{\epsilon}{\lambda}
$$

and $\lim \sup _{n}\left\|\left(\lambda R_{\lambda}\right)^{n}\right\| / n \leq \epsilon / \lambda$. Let $\epsilon \rightarrow 0$ to conclude the proof.

Lemma 2。If $\mu R_{\mu} x=x$ for some $\mu>0$, then $T_{t} x=x$ for every $t>0$.

Proof. By the resolvent equation $\left(R_{\lambda}-R_{\mu}\right) x=(\mu-\lambda) R_{\lambda} R_{\mu} x$.

$$
R_{\lambda} x-R_{\mu} x=R_{\lambda} \mu R_{\mu} x-\lambda R_{\lambda} R_{\mu} x=R_{\lambda} x-\lambda R_{\lambda} R_{\mu} x \text {. }
$$

Thus

$$
\left(I-\lambda R_{\lambda}\right) x=\mu\left(I-\lambda R_{\lambda}\right) R_{\mu} x=0,
$$

and $\lambda R_{\lambda} x=x$ for every $\lambda>0$. The lemma follows from the inversion formula $(11.7 .2)$ in [5].

Remark. If $\sup _{t \geq 0}\left\|T_{t}\right\| \leq M$, we may assume $M=1$. Then Lemma 1 is trivial, and Lemma 2 is proved by Falkowitz [3] without using the inversion formula.

Proof of the Theorem. We denote by $R(A)$ the range of $A$ and by $Y$ the closure of $R(A)$.

(2) $\Rightarrow$ (3). For $\lambda>0(\lambda I-A) R_{\lambda}=I$ and for $x \in D(A), R_{\lambda}(\lambda I-A) x=x$ [2, Lemma VIII.1.7]. Hence $\lambda R_{\lambda}=I+A R_{\lambda}$ and $\left(\lambda R_{\lambda}-I\right) X \subset R(A)$. By (2) $Y=R(A)$, so that for $y \in Y$ there is an $x \in D(A)$ with $A x=y$, and

$$
x=R_{\lambda}(\lambda I-A) x=\lambda R_{\lambda} x-R_{\lambda} y \quad \text { or } \quad R_{\lambda} y=\left(\lambda R_{\lambda}-I\right) x .
$$

Thus

$$
y=(\lambda I-A) R_{\lambda} y=(\lambda I-A)\left(\lambda R_{\lambda}-I\right) x=\left(\lambda R_{\lambda}-I\right)(\lambda I-A) x
$$

and $Y=\left(\lambda R_{\lambda}-I\right) X$. Thus by applying the uniform ergodic theorem of [6] to the operator $\lambda R_{\lambda}$ we obtain (3), since $\left\|\left(\lambda R_{\lambda}\right)^{n}\right\| / n \rightarrow 0$ by Lemma 1 .

$(2) \Rightarrow(1)$. For $x \in D(A), A T_{t} x=T_{t} A x$ and therefore $T_{t} Y \subset Y$. The generator $A_{1}$ of the restriction of $\left\{T_{t}\right\}$ to $Y$ will be the restriction of $A$ to $Y \cap D(A)$. It is shown above that (2) implies $Y=\left(I-\lambda R_{\lambda}\right) X$, and the uniform ergodic theorem shows that $I-\lambda R_{\lambda}$ is invertible on $Y$. If $A_{1} y=0$ 
for $y \in Y \cap D(A)$, then

$$
y=R_{\lambda}(\lambda I-A) y=\lambda R_{\lambda} y \quad \text { or } \quad\left(I-\lambda R_{\lambda}\right) y=0,
$$

and $y=0$. Thus $A_{1}$ is one-to-one. On $Y$ we have, as above, $\left(I-\lambda R_{\lambda}\right) Y \subset$ $R\left(A_{1}\right)$. But from $(2) \Rightarrow(3)$ we have

$$
Y \supset R\left(A_{1}\right) \supset\left(I-\lambda R_{\lambda}\right) Y=\left(I-\lambda R_{\lambda}\right) X=Y=R(A),
$$

and $R\left(A_{1}\right)=Y$, so $A_{1}^{-1}$ is defined for all of $Y . A_{1}$ is closed, therefore $A_{1}^{-1}$ is closed, and by the closed graph theorem $A_{1}^{-1}$ is continuous. For $y \in Y=$ $R\left(A_{1}\right)$ there is an $x \in D(A) \cap Y$ such that $A x=y$ and $\|x\| \leq\left\|A_{1}^{-1}\right\|\|y\|$. By [2, Lemma VIII.1.7]

$$
\left(T_{t}-I\right) x=\int_{0}^{t} T_{r} A x d r=\int_{0}^{t} T_{r} y d r
$$

and $\left\|t^{-1} \int_{0}^{t} T_{r} y d r\right\| \leq\left\|A_{1}^{-1}\right\|\left(\left\|T_{t}\right\|+1\right) t^{-1}\|y\|$. Hence on $Y$ we have uniform convergence to 0 . But $X=Y \oplus\left\{x: R_{1} x=x\right\}$ by the uniform ergodic theorem, Lemma 2 then yields that $X=Y \oplus\left\{x: T_{t} x=x, \forall t>0\right\}$, and (1) follows immediately.

$(3) \Rightarrow(2)$. By the uniform ergodic theorem $\left(I-R_{1}\right) X$ is closed. In $(2) \Rightarrow(3)$ we have shown $\left(I-R_{1}\right) X=R(A)$ and $R(A)$ is closed.

$(1) \Rightarrow(2)$. It follows from (1) that $E^{2}=E$, with $E X=\left\{x: T_{t} x=\right.$ $x, \forall t \geq 0\}$. As shown above, $T, Y \subset Y$. For $x \in D(A)$, we have by [2, Lemma VIII.1.7] that $\left(T_{t}-I\right) x=\int_{0}^{t} T_{r} A x d r \in Y$. Since $D(A)$ is dense, we have that $c \operatorname{lm} \bigcup_{t \geq 0}\left(I-T_{t}\right) X \subset Y$. On the other hand, for $x \in D(A), A x=$ $\lim _{h \rightarrow 0} h^{-1}\left(T_{h}-I\right) x$, so that $Y \subset c \operatorname{lm} \bigcup_{t \geq 0}\left(I-T_{t}\right) X$ and equality holds. Hence by (1), $X=E X \oplus Y$ (see [2, VIII.7.2]). Thus by restricting ourselves to $Y$ we have $Y=\overline{R\left(A_{1}\right)}$ (where $A_{1}$ is the generator of the restriction, with domain $D(A) \cap Y)$. Thus we may and do assume $X=Y$, and $\left\|t^{-1} \int_{0}^{t} T_{s} d s\right\| \rightarrow 0$.

Let $x \in D(A)$ satisfy $A x=0$. Then $x=R_{1}(I-A) x=R_{1} x$ and by Lemma $2 T_{t} x=x$ for every $t \geq 0$. Since $X=Y, x=0$ and $A$ is one-to-one, with $A^{-1}$ defined on $R(A)$. For fixed $t$ large enough, $\left\|t^{-1} \int_{0}^{t} T_{r} d r\right\|<1$ and $I-t^{-1} \int_{0}^{t} T_{r} d r$ is invertible, and so is $\int_{0}^{t}\left(T_{r}-I\right) d r$. For $y \in R(A)$ take $x \in D(A)$ with $A x=y$. Then

and

$$
\int_{0}^{t}\left(T_{s}-I\right) x d s=\int_{0}^{t}\left(\int_{0}^{s} T_{r} A x d r\right) d s=\int_{0}^{t}\left(\int_{0}^{s} T_{r} y d r\right) d s
$$

$$
\|x\|=\left\|A^{-1} y\right\| \leq\left\|\left(\int_{0}^{t}\left(T_{s}-I\right) d s\right)^{-1}\right\|\left\|\int_{0}^{t}\left(\int_{0}^{s} T_{r} y d r\right) d s\right\| \leq K\|y\| .
$$


Hence $A^{-1}$ is continuous with dense domain (in $X=Y$ ). Since $A$ is closed, $A^{-1}$ is defined on all of $Y$, and $Y=R\left(A_{1}\right) \subset R(A)$, so that $R(A)=Y$ is closed.

$(1) \Rightarrow(4)$. We have just seen above that $X=E X \oplus R(A)$, with $E X=$ $\left\{x: T_{t} x=x, t \geq 0\right\}$, so that we have to show only $\lambda R_{\lambda} \rightarrow 0$ uniformly on $Y=$ $R(A)=R\left(A_{1}\right)$. Again we may and do assume $X=Y$, and $A^{-1}$ is continuous. Let $0<\lambda \leq \delta<1 /\left\|A^{-1}\right\|$. For $y \in Y$

$$
\left\|\lambda R_{\lambda} y\right\|=\left\|\lambda R_{\lambda} A A^{-1} y\right\| \leq\left\|\lambda\left(\lambda R_{\lambda}-I\right)\right\|\left\|A^{-1}\right\|\|y\| \text {. }
$$

Hence for $0<\lambda \leq \delta$ we have,

$$
\left\|\lambda R_{\lambda}\right\| \leq\left(1+\left\|\lambda R_{\lambda}\right\|\right) \delta\left\|A^{-1}\right\|, \quad \text { or } \quad\left\|\lambda R_{\lambda}\right\| \leq \delta\left\|A^{-1}\right\| /\left(1-\delta\left\|A^{-1}\right\|\right) \equiv M .
$$

Now we have

$$
\left\|\lambda R_{\lambda}\right\| \leq\left\|\lambda\left(\lambda R_{\lambda}-I\right)\right\|\left\|A^{-1}\right\| \leq \lambda(1+M)\left\|A^{-1}\right\| \underset{\lambda \rightarrow 0^{+}}{\rightarrow} 0 .
$$

(4) $\Rightarrow$ (2). If $y=A x$ for $x \in D(A)$, then $\lambda R_{\lambda} y=\lambda R_{\lambda} A x=\lambda\left(\lambda R_{\lambda}-I\right) x$ $\rightarrow_{\lambda \rightarrow 0^{+}} 0$. Restricting ourselves to $Y$ we have $\left\|\lambda R_{\lambda}\right\|_{Y} \rightarrow 0$. But on $Y$ we have for $\lambda>0$ small enough that $I-\lambda R_{\lambda}$ is invertible, hence

$$
Y=\left(I-\lambda R_{\lambda}\right) Y \subset\left(I-\lambda R_{\lambda}\right) X=R(A)
$$

(see $(2) \Rightarrow(1)$ for last equality) and $R(A)=Y$.

Remark. [5, Theorem 18.8.4] treats more general semigroups, but is only concerned with Abel convergence, and does not include the sufficiency of $R(A)$ being closed. The method presented here is different, not using the operational calculus and spectral theory used in [5].

Corollary 1. Let $\left\{T_{t}\right\}$ be as above. The following two conditions are equivalent:

(1) $t^{-1} \int_{0}^{t} T_{r} d r$ converges uniformly as $t \rightarrow \infty$.

(2) There is a $\delta>0$ such that $\sup _{0<\lambda \leq \delta}\left\|R_{\lambda} y\right\|<\infty$ for every $y \in \overline{R(A)}$.

If $\left\|T_{t}\right\| \leq M$ for $t \geq 0$, then the following (sufficient) condition is also equivalent to the previous two:

(3) $\sup _{t \geq 0}\left\|\int_{0}^{t} T_{r} y d r\right\|<\infty$ for every $y \in \overline{R(A)}$.

Proof. $(1) \Rightarrow(2)$. By the Theorem $R(A)$ is closed. For $y \in R(A)$ we have $x \in D(A)$ with $A x=y$, and $R_{\lambda} y=R_{\lambda} A x=-x+\lambda R_{\lambda} x$. The proof of (1) $\Rightarrow$ (4) in the Theorem shows that $\sup _{0<\lambda \leq \delta}\left\|\lambda R_{\lambda}\right\|<\infty$, and (2) follows. (In fact, $\sup _{0<\lambda<\infty}\left\|R_{\lambda} y\right\|<\infty$ : by the computations of Lemma 1 , $\sup _{\delta \leq \lambda}\left\|\lambda R_{\lambda}\right\|<\infty_{\text {.) }}$ 
$(2) \Rightarrow(1)$. We look at the semigroup restricted to $Y=\overline{R(A)}$, with generator $A_{1}$. By the principle of uniform boundedness, $\sup _{0<\lambda \leq \delta}\left\|R_{\lambda}\right\|_{Y}<\infty$ and therefore $\lim _{\lambda \rightarrow 0}\left\|\lambda R_{\lambda}\right\|_{Y}=0$. By the Theorem, $Y=R\left(A_{1}\right) \subset R(A)$. Hence $R(A)$ is closed and (1) follows from the Theorem.

(3) $\Rightarrow(1)$ is proved similarly.

If $\left\|T_{t}\right\| \leq M$ for every $t \geq 0$ and we assume (1), then for $y=A x(x \in D(A))$ we have, by [2, Lemma VIII.1.7],

$$
\left\|\int_{0}^{t} T_{r} y d r\right\|=\left\|\int_{0}^{t} T_{r} A x d r\right\|=\left\|\left(T_{t}-I\right) x\right\| \leq(M+1)\|x\| .
$$

Since $R(A)$ is closed, (3) follows.

Corollary 2. Assume $\left\|T_{t}\right\| \leq M$ for every $t \geq 0$. Let $\nu$ be a probability measure on $[0, \infty), \nu(\{0\})<1$, and define $U x=\int T_{t} x d \nu$. If $N^{-1} \Sigma_{n=0}^{N-1} U^{n}$ converges uniformly, then $t^{-1} \int_{0}^{t} T_{r} d r$ converges uniformly (as $t \rightarrow \infty$ ).

Proof. Since $\|x\|=\sup _{t \geq 0}\left\|T_{t} x\right\|$ is an equivalent norm for $X$ such that $\left\|T_{t}\right\| \leq 1$, we may and do assume $\left\|T_{t}\right\| \leq 1$.

Note that $U$ is well defined in the strong operator topology, by [5, Theorem 3.7.4].

By the discrete uniform ergodic theorem $X=(I-U) X \oplus\{x: U x=x\}$; since $T_{t}$ commutes with $U$, both subspaces are invariant under $\left\{T_{t}\right\}$, and we can restrict ourselves to each one. Hence it suffices to treat two cases:

(1) $I-U$ is invertible. (2) $U=I$.

Case 1. Fix $\epsilon>0$. Let $\beta>0$ be such that $\nu([0, \beta])>1-\epsilon$. Define $\nu_{\epsilon}(C)=\nu(C \cap[0, \beta]) / \nu([0, \beta])$. Then $\left\|\nu-\nu_{\epsilon}\right\| \leq 1 /(1-\epsilon)-1+\epsilon$. Define $U_{\epsilon} x=\int T_{t} x d \nu_{\epsilon}$. Then

$$
\left\|(I-U)-\left(I-U_{\epsilon}\right)\right\|=\left\|U-U_{\epsilon}\right\| \leq\left\|\nu-\nu_{\epsilon}\right\| \underset{\epsilon \rightarrow 0}{\rightarrow} 0
$$

and $I-U_{\epsilon}$ is also invertible for $\epsilon$ small enough. Thus we may and do assume that $\nu$ is supported on a finite interval $[0, \beta]$.

Let $A$ be the infinitesimal generator of $\left\{T_{t}\right\}$. Since $D(A)$ is dense, for $y \in X$ there is a sequence $x_{n} \in D(A)$ with $x_{n} \rightarrow(I-U)^{-1} y$. Hence

$$
y=\lim _{n \rightarrow \infty}(I-U) x_{n}=\lim _{n \rightarrow \infty} \int\left(I-T_{t}\right) x_{n} d \nu=-\lim _{n \rightarrow \infty} \int\left(\int_{0}^{t} T_{r} A x_{n} d r\right) d \nu,
$$

which shows that $y \in \overline{R(A)}$ (since $\overline{R(A)}$ is invariant under $\left\{T_{t}\right\}$ ). Let $z_{n} \in D(A)$ satisfy $A z_{n} \rightarrow y$. Then 


$$
\begin{aligned}
\left\|(I-U) z_{n}-(I-U) z_{m}\right\| & =\left\|\int\left\{\int_{0}^{t} T_{r}\left(A z_{n}-A z_{m}\right) d r\right\} d \nu\right\| \\
& \leq \int\left\{\int_{0}^{t}\left\|A z_{n}-A z_{m}\right\| d r\right\} d \nu \\
& =\left\|A z_{n}-A z_{m}\right\| \int t d \nu \leq \beta\left\|A z_{n}-A z_{m}\right\| .
\end{aligned}
$$

Thus $\left\{(\bar{I}-U) z_{n}\right\}$ is a Cauchy sequence, and $z_{n}=(I-U)^{-1}(I-U) z_{n}$ converges (strongly), say to $z$. Then $z_{n} \rightarrow z$ and $A z_{n} \rightarrow y$, and since $A$ is a closed operator, $z \in D(A)$ and $y=A z$. Thus $X=R(A)$ and by the theorem $\left\|t^{-1} \int_{0}^{t} T_{r} d r\right\| \rightarrow 0$.

Case 2. We show first that $U=I$ implies $T_{t_{0}}=I$ for some $t_{0}>0$. If, for $t_{0}>0, a=\nu\left(\left\{t_{0}\right\}\right)>0$, then for every $x \in X$ we have $x=U x=\alpha T_{t_{0}} x+$ $(1-a) \int T_{t} x d \nu_{1}$, where $\nu_{1}=\left(\nu-a \delta_{t_{0}}\right) /(1-a)$ with $\delta_{t_{0}}$ the Dirac measure at $t_{0}$. By [3, Lemma 1], $T_{t_{0}} x=x$, and thus $T_{t_{0}}=I$. Assume now that $\nu(\{t\})=0$ for $t>0$. Let $F(t)=\nu((-\infty, t])$ be the distribution of $\nu$. Then $F(t)$ is continuous at $t>0$, and since $F(0)=\nu(\{0\})<1$, there is a $t^{\prime}>0$ with $0<F\left(t^{\prime}\right)=a<1$. Let $t_{0}=\sup \{t: F(t)=a\}$, and $F\left(t_{0}\right)=a$ by continuity. Take $t_{0}<t_{n} \downarrow t_{0}$, and let $a_{n}=\nu\left(\left[t_{0}, t_{n}\right]\right)$. Define (since $1>a_{n}>0$ )

$$
\nu_{n}(C)=\nu\left(C \cap\left[t_{0}, t_{n}\right]\right) / a_{n} \text { and } \mu_{n}(C)=\left\{\nu(C)-a_{n} \nu_{n}(C)\right\} /\left(1-a_{n}\right) .
$$

Then $\nu_{n}$ and $\mu_{n}$ are nonzero probability measures with $a_{n} \nu_{n}+\left(1-a_{n}\right) \mu_{n}=$ $\nu$. By [3, Lemma 1] we also have that $\int T_{t} x d \nu_{n}=x$ for every $x \in X$, so that $\int T_{t} d \nu_{n}=I$. Fix $x \in X, x^{*} \in X^{*}$. Then $\left\langle x^{*}, T_{t} x\right\rangle$ is continuous on $\left[t_{0}, t_{n}\right]$ and there is a point $t_{0} \leq s_{n} \leq t_{n}$ such that $\left\langle x^{*}, T_{s_{n}} x\right\rangle=$ $\int\left\langle x^{*}, T_{t} x\right\rangle d \nu_{n}=\left\langle x^{*}, x\right\rangle$. Since also $\lim s_{n}=t_{0}$, the continuity of $\left\{T_{t}\right\}$ yields $\left\langle x^{*}, T_{t_{0}} x\right\rangle=\left\langle x^{*}, x\right\rangle$. Hence $T_{t_{0}}=I$. To finish the proof in Case 2, denote $n=\left[t / t_{0}\right]$. Then for $t>t_{0}$ we have (with $\alpha=t / t_{0}-n$ )

$$
\begin{aligned}
t^{-1} \int_{0}^{t} T_{r} d r & =t^{-1} \int_{0}^{n t_{0}} T_{r} d r+t^{-1} \int_{n t_{0}}^{t} T_{r} d r \\
& =t^{-1} n \int_{0}^{t_{0}} T_{r} d r+t^{-1} T_{n t_{0}} \int_{0}^{a t_{0}} T_{r} d r \rightarrow t_{0}^{-1} \int_{0}^{t_{0}} T_{r} d r .
\end{aligned}
$$

Remarks. (1) The corollary can be applied if $I-U-Q$ is invertible for some compact operator $Q$ (see [6]). (2) In the discrete case $\left\{T^{n}\right\}$ treated in [6] there is a requirement $\sum_{i=1}^{k} a_{i}=1$. It can be removed by an approxima- 
tion argument, as in the proof of Corollary 2. (Also see the example in the appendix.)

Appendix. We show here that if $\left\|T^{n} / n\right\| \rightarrow 0$, then Abel and Cesàro convergence of $\left\{T^{n}\right\}$ are equivalent in the uniform operator topology. Hille [4] proved it for the case sup $\left\|T^{n}\right\|<\infty$, using a general method which does not apply in the case that $T$ is not power-bounded. (An extension of Hille's result in the strong operator topology is a consequence of [1, Theorem 1].)

Proposition. Let $T$ be a linear operator on $X$ with $\left\|T^{n} / n\right\| \rightarrow 0$. Then the following conditions are equivalent:

(1) $N^{-1} \Sigma_{n=0}^{N-1} T^{n}$ converges in the uniform operator topology.

(2) $\lim _{r \rightarrow 1^{-}}\left\|(1-r) \sum_{n=0}^{\infty} r^{n} T^{n}-E\right\|=0$ for some operator $E$.

(3) $(I-T) X$ is closed.

Proof. Let $Y=\overline{(I-T) X}$, which is invariant under $T$, and let $S$ on $Y$ be the restriction of $T$.

$(1) \Rightarrow(2)$. Since this follows from Hille's general results [4], we only sketch a simple proof for the present situation. From (1) we have $X=Y \oplus$ $\{x: T x=x\}$, and we have only to show $\left\|(1-r) \sum_{n=0}^{\infty} r^{n} S^{n}\right\| \rightarrow 0$. But by the uniform ergodic theorem, $I-S$ is invertible on $Y$. Thus, for $0<r<1$,

$$
\begin{aligned}
\left\|(1-r) \sum_{n=0}^{\infty} r^{n} S^{n}\right\| & =\left\|(1-r) \sum_{n=0}^{\infty} r^{n} S^{n}(I-S)(I-S)^{-1}\right\| \\
& \leq\left\{\left\|(1-r) \sum_{n=1}^{\infty}\left(r^{n}-r^{n-1}\right) S^{n}\right\|+(1-r)\right\}\left\|(I-S)^{-1}\right\| \\
& \leq(1-r)\left\|(I-S)^{-1}\right\|\left\{1+(1-r) \sum_{n=1}^{\infty} r^{n-1}\left\|S^{n}\right\|\right\} .
\end{aligned}
$$

Fix $\epsilon>0$. For $n>k,\left\|S^{n}\right\| / n<\epsilon$ and

$$
\begin{aligned}
(1-r)^{2} \sum_{n=1}^{\infty} r^{n-1}\left\|S^{n}\right\| & \leq(1-r)^{2} \sum_{n=1}^{k} r^{n-1}\left\|S^{n}\right\|+\epsilon(1-r)^{2} \sum_{n=1}^{\infty} r^{n-1} n \\
& =(1-r)^{2} \sum_{n=1}^{k} r^{n-1}\left\|S^{n}\right\|+\epsilon \underset{r \rightarrow 1-}{\rightarrow} \epsilon .
\end{aligned}
$$

Let $\epsilon \rightarrow 0$ to conclude (2).

(2) $\Rightarrow$ (3). We shall show $(I-S) Y=Y$, which implies (3). Hence we restrict ourselves to $Y$. If $y=(I-T) x$, then 


$$
\begin{aligned}
(1-r) \sum_{n=0}^{\infty} r^{n} S^{n} y & =(1-r) \sum_{n=0}^{\infty} r^{n}(I-T) T^{n} x \\
& =(1-r) \sum_{n=0}^{\infty} r^{n} T^{n} x-(1-r) r^{-1} \sum_{n=0}^{\infty} r^{n} T^{n} x+(1-r) r^{-1} x \\
& \rightarrow E x-E x+0=0 .
\end{aligned}
$$

Hence $\left\|(1-r) \sum_{n=0}^{\infty} r^{n} S^{n}\right\| \rightarrow_{r \rightarrow 1^{-}} 0$. Thus for fixed $0<r<1$, close enough to $1, I-(1-r) \Sigma_{n=0}^{\infty} r^{n} S^{n}$ is invertible. Since $\sum_{n=0}^{\infty} r^{n} S^{n}$ is defined in the uniform operator topology (by virtue of $\left\|T^{n}\right\| / n \rightarrow 0$ ), we have that

$$
(1-r) \sum_{n=1}^{\infty} r^{n}\left(\sum_{i=0}^{n-1} S^{i}\right)=(1-r) \sum_{i=0}^{\infty}\left(\sum_{n=i+1}^{\infty} r^{n}\right) S^{i}=\sum_{i=0}^{\infty} r^{i+1} S^{i}
$$

is also defined in the uniform operator topology. But

$$
I^{\circ}-(1-r) \sum_{n=0}^{\infty} r^{n} S^{n}=(1-r) \sum_{n=0}^{\infty} r^{n}\left(I-S^{n}\right)=(I-S)(1-r) \sum_{n=1}^{\infty} r^{n}\left(\sum_{i=0}^{n-1} S^{i}\right)
$$

and $(I-S) Y=Y$ by invertibility of the left-hand side.

$(3) \Longrightarrow(1)$ is proved in [6].

Example. We exhibit an operator $T$ satisfying $\|T\| \leq 1,(I-T) X=X$ and $\left(I-T^{2}\right) X$ is not closed. Since $\left\|N^{-1} \Sigma_{n=0}^{N-1} T^{n}\right\| \rightarrow 0, T$ satisfies the conditions of $[6$, Corollary 2$]$ (with $Q=0$ ), but is not quasi-compact (since this implies $T^{k}$ are all quasi-compact).

Let $X=l_{2}, 0>\lambda_{1}>\lambda_{2}>\cdots>\lambda_{n}>\downarrow-1$. Define $T x=\left\{\lambda_{i} x_{i}\right\}$ where $x=\left\{x_{i}\right\}$. Then $(I-T)^{-1} x=\left\{x_{i} /\left(1-\lambda_{i}\right)\right\},\left(I-T^{2}\right) X$ contains all sequences with finitely many nonzero terms, and is dense. But $-1 \in \sigma(T)$ and $I+T$ is not invertible, but $I+T$ is one-to-one. Hence,

$$
\left(I-T^{2}\right) X \subset(I+T) X \neq X=\overline{\left(I-T^{2}\right) X} .
$$

\section{REFERENCES}

1. A. Brunel, H. Fong and L. Sucheston, An ergodic super-property of Banach spaces, defined by a class of matrices (to appear).

2. N. Dunford and J. T. Schwartz, Linear operators. I: General theory, Pure and Appl. Math., vol. 7, Interscience, New York, 1958. MR 22 \#8302.

3. M. Falkowitz, On finite invariant measures for Markov operators, Proc. Amer. Math. Soc. 38 (1973), 553-557. MR 47 \#880.

4. E. Hille, Remarks on ergodic theorems, Trans. Amer. Math. Soc. 57 (1945), 246-269. MR 6, 276. 
5. E. Hille and R. S. Phillips, Functional analysis and semi-groups, rev. ed., Amer. Math. Soc. Colloq. Publ., vol. 31, Amer. Math. Soc., Providence, R. I., 1957. MR 19, 664 .

6. M. Lin, On the uniform ergodic theorem, Proc. Amer. Math. Soc. 43 (1974), $337-340$.

DEPARTMENT OF MATHEMATICS, OHIO STATE UNIVERSITY, COLUMBUS, OHIO 43210 